\title{
Simulation of the dynamic processes of a low- capacity combine harvester movement
}

\author{
Zakhid Godzhaev ${ }^{1 *}$, Sergey Senkevich ${ }^{1}$, Victor Kuzmin ${ }^{1}$, Ekaterina Ilchenko ${ }^{1}$, Mikhail \\ Chaplygin $^{1}$, Ilya Alekseev ${ }^{1}$, and Alexander Prilukov ${ }^{1}$ \\ ${ }^{1}$ Federal Scientific Agroengineering Center VIM, 1st Institute pas. 5, Moscow, 109428, Russia
}

\begin{abstract}
To automate the process of harvesting crops, different types of harvesting machines are required. The most common type of machines used to automate ingathering are harvesters. The use of harvesters for collecting grain crops is rational in fields from 2 hectares. On smaller areas it is not profitable and difficult to apply. For applications in small areas, low-capacity harvesters with a throughput of the thresher up to $1 \mathrm{~kg} / \mathrm{s}$ (small-sized) may be suitable. The purpose of this study is to analyze the cushioning mass control system of a low-capacity unmanned combine harvester using computer simulation, as well as the simulation of directional stability and turning. To calculate vertical vibrations in the Matlab/Simulink software package, a model of a cushioning system for a wheeled agricultural combine was prepared. In the same software package, simulation of directional stability and turning implementation was carried out. The parameters of vibration displacement, vibration velocity and acceleration, as well as the vibration frequency indicators on the operator's seat are determined. Comparison of the simulation results of the initial and corrected direction of movement of the harvester showed that for this model the maximum deviation from the planned path is a maximum of $10 \%$, which is within acceptable limits. The maximum deviation of the harvester from the course does not exceed the permissible values, which is acceptable accuracy to ensure directional stability.
\end{abstract}

\section{Introduction}

The process of harvesting crops has its own characteristics and difficulties that require its automation in order to increase the productivity of the harvesting unit. The most common way to automate ingathering is to use harvesters. For wheat and rice, the use of harvesters is rational with the area of the cultivated plot of land starting from 2 hectares [1]. This is due to both economic and ergonomic reasons - in addition to monetary costs for the purchase and maintenance of the machine, the dimensions of the harvester prevent the use of it in small areas. A large machine is not able to move and rotate freely in small areas. However, small areas also require automation. There are a large number of studies aimed at creating small-sized agricultural robots capable of ingathering various types of crops, watering plants, spraying with chemicals, etc. For example, there is the concept of a modular

*Corresponding author: fic51@,mail.ru 
agricultural robot, parts of which can be replaced depending on production needs [2]. It is a tractor with a trolley, on which a robot manipulator is located, which is able, for example, to collect fruits from trees. But most of the development of agricultural robots are aimed at use in enclosed spaces (greenhouses) or have an extremely narrow specialization. The conditions of use dictate to such robots a specific set of components, ranging from the chassis to the method of orientation in space, which makes it impossible to use them on the field. Small combines could be suitable for these purposes.

In the agricultural field, a lot of research has been done using mathematical modeling methods. So, for example, a model of the area of influence on tractor tracks was developed using kinematics and geometry for various equipment and work scenarios [3]. During its development, it was found out that the pressure on the soil depends on the distance between the tracks or the wheels, their area, the size of the area, and the weight of the machine and equipment. Although the tracked undercarriage system has certain advantages when moving along the ground, it also has a number of disadvantages, including: less maneuverability, massiveness, and a specific shape that must be taken into account when harvesting certain types of crops.

For combines of low-capacity, the wheel undercarriage system is more convenient. However, it also needs to be correctly selected based on certain soil characteristics. There are many tire models that are presented by authors in [4,5]. In these works, a very accurate tire model is described that describes their behavior in the contact area of the tire with the ground for both longitudinal and lateral stiffness of the tire. This model may be applicable when considering traction and steering maneuvers. For maneuvering, it is also necessary to have a good braking system to ensure the safety of both humans and products [6]. Their method, excluding traditional field trials, provides an effective but simple way to design and evaluate the brake system of the chassis of combine harvesters. Following the example of the author of [7], it is possible to test the harvester to determine the optimal velocity of movement during ingathering.

The most important component of the harvester is the control and navigation system. There are a lot of orientation methods, as well as components for these purposes, and to choose the optimal control method, one must proceed from the existing conditions. One of the field-acceptable methods of orientation in space is terrain mapping, which is an important component of the introduction of accurate farming methods [8]. Promising is the use of an autonomous control system based on laser scanners that can navigate the terrain in real time [9].

Of no small importance in the control system is the maintenance of operability and control over the performance of the work of individual elements of the system. This requires a more in-depth study and modernization of the system in accordance with the latest technology. So the processes in the control system should not be interrupted due to power supply problems, for example, due to power fluctuations in the power sources, therefore, the equipment should be able to function correctly with unpredictable and variable supply voltages. To meet this need, a neural network was developed, which is described by a team of authors in [10]. You should also pay attention to control of directional stability [11] and velocity of movement [12]. Not only one combine harvester can work simultaneously on the field, but it is also possible to use several harvesters, and, therefore, a monitoring and control system designed for group interaction is needed $[12,13$, $14,15]$. Each control unit must be directly located at each harvester with an active drive, with which technological operations are performed [16]. Combine harvester management can be improved with the help of improved aggregates and units, including in the field of autonomous power supply $[17,18]$, as well as mathematical modeling of harvester control processes [19].

Based on the review of scientific research, we can conclude that: 
1. When considering the selection of the most suitable direction for the development of a combine harvester control system, most researchers adhere to the direction of a mixed control system - the use of satellite navigation, base stations for correcting navigation signals and machine vision;

2. Among the technical characteristics of control systems that are developed by many researchers include:

- autonomous movement in compliance with directional stability during harvesting;

- autonomous u-shaped turns at the end of the field;

- autonomous operation of the combine reaper in unmanned mode based on field conditions;

- ensuring the possibility of working the developed sample at night.

\section{Purpose of the study}

The purpose of this research study is to justify the work on creating a movement control system for an unmanned combine harvester with a capacity of up to $1 \mathrm{~kg} / \mathrm{s}$, according to the technical task of the customer - the Ministry of Education and Science of Russia, using computer simulation of directional stability and turning implementation of the harvester.

\section{Materials and methods}

To carry out this work, a mathematical modeling method was applied and the Matlab/Simulink software package was used.

\subsection{Simulation of the cushioning system of the wheel harvester}

To calculate vertical vibrations in the Matlab/Simulink software package, a model of a cushioning system for a low-capacity wheeled agricultural combine harvester was prepared. The mass of the harvester is brought to its center of mass, which made it possible to simplify the model of the cushioning system. The single-mass oscillatory system of the harvester was considered using the D'Alembert-Lagrange equation, according to which the sum of the work of ideal connections on any virtual movement of the system is zero:

where,

$$
\delta A_{\Sigma}=\Sigma F \times \delta r=0
$$

$A_{\Sigma}$ - work of the total reduced moment to its possible movement;

$F$ - force acting on the center of mass of the system;

$\delta r$ - perfect movement.

First, we will reduce all the vibrational masses of the combine to a single-mass system. The transformation equations are given in equations (2)-(5).

where:

$$
\mathrm{M}_{\Sigma}^{\mathrm{red}} \times d \varphi_{1}=\sum F_{i} \times d s_{i}+\sum \mathrm{M}_{i} \times d \varphi_{i}
$$

$\mathrm{M}_{\Sigma}^{\text {red }}$ - reduced total torque acting on the center of mass of the system;

$F_{i}$ - force acting on the center of mass of the system;

$\mathrm{M}_{i}$ - i-th moment of forces acting on the center of mass of the system;

$\varphi_{\mathrm{M}}$ - change in the angle of displacement of the center of mass of the system;

$v_{i}$ - linear velocity of displacement of the center of mass of the system;

$\varphi_{\mathrm{M}}=\varphi_{1}=\varphi_{i}$.

Applying the operation of multiplying the left and right sides by $\frac{1}{d t}$ in equation (2), and 
applying the simplest transformations, taking $\frac{d s}{d t}=v \cup \frac{d \varphi}{d t}=\omega$ into account, we obtain equation (3)

where:

$$
\mathrm{M}_{\Sigma}^{\mathrm{red}} \times \omega_{\mathrm{M}}=\sum F_{i} \times v_{i} \times \cos \left(F_{i}^{\wedge} v_{i}\right)+\sum \mathrm{M}_{i} \times \omega_{i}
$$

$\omega_{\mathrm{M}}-$ angular velocity of displacement of the center of mass of the system;

$v_{i}$ - linear velocity of displacement of the center of mass of the system;

$\omega_{i}$ - angular velocity of displacement of the center of mass of the system;

$v_{i}$ - linear velocity of displacement of the center of mass of the system.

or

$$
\mathrm{M}_{\Sigma}^{\mathrm{red}}=\sum F_{i} \times \frac{v_{i}}{\omega_{\mathrm{M}}} \times \cos \left(F_{i}^{\wedge} v_{i}\right)+\sum \mathrm{M}_{i} \times \frac{\omega_{i}}{\omega_{\mathrm{M}}}
$$

where:

$$
\mathrm{M}_{\Sigma}^{\mathrm{red}}=\sum F_{i} \times v_{q i} \times \cos \left(F_{i}^{\wedge} v_{i}\right)+\sum \mathrm{M}_{i} \times u_{i-1}
$$

$\frac{v_{i}}{\omega_{\mathrm{M}}}=v_{q i}-$ gear function;

$\frac{\omega_{i}}{\omega_{\mathrm{M}}}=u_{i-1}-$ gear ratio.

Therefore, reducing the reduced mass to the center of mass of the object of study and applying the D'Alembert-Lagrange law, we have the following equation of motion of the control object:

$$
\mathrm{m}_{\mathrm{pm}} \times \mathrm{z}_{1}-2 \times \mathrm{C}_{\mathrm{sh}} \times\left(\mathrm{z}_{1}-\mathrm{q}_{1}\right)-2 \times \mathrm{k}_{\mathrm{sh}} \times\left(\mathrm{z}_{1}-\mathrm{q}_{1}\right)=0
$$

where $k_{s h}=\frac{\gamma \times C_{s h}}{f}-$ damping coefficient of the system;

$C_{s h}$ - coefficient of rigidity of the cushioning system;

$\gamma$ - system attenuation decrement;

$f$-equivalent oscillation frequency of the system.

The motion model of the harvester, according to the calculation scheme described above, is represented using the Matlab/Simulink software package (Figure 1).
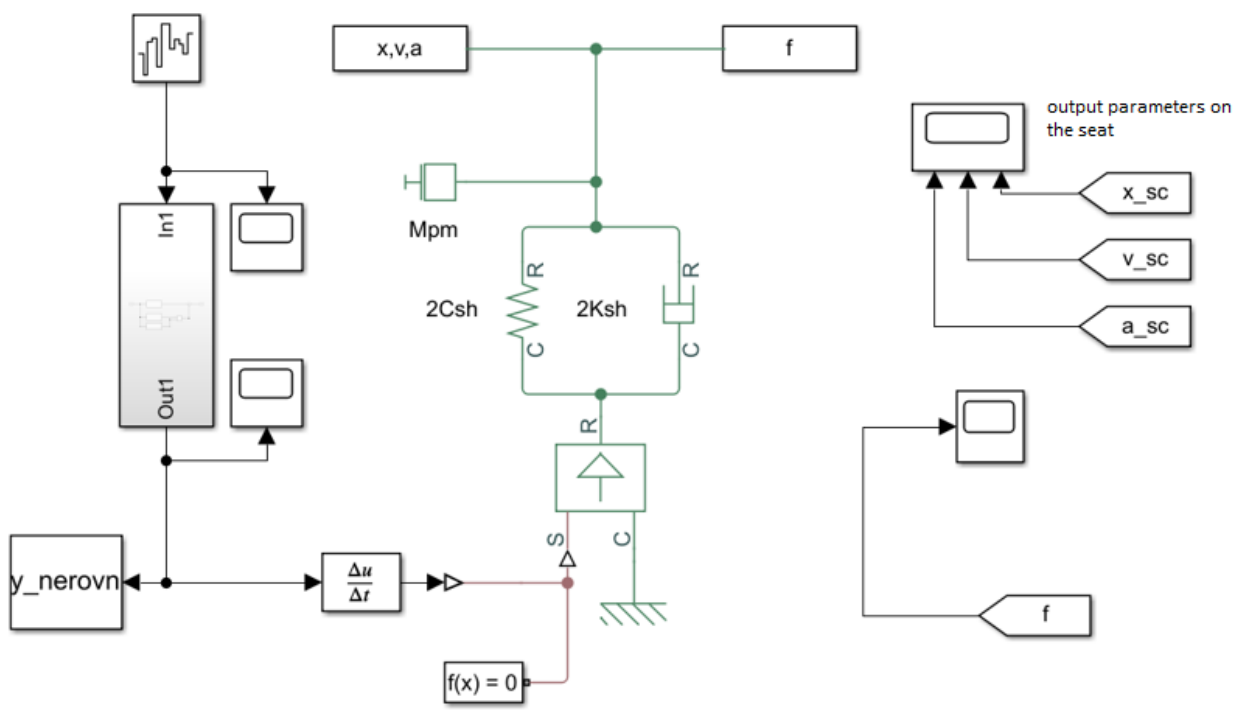

Fig. 1. The model of the cushioning system of the harvester

The model consists of 3 blocks:

- block generating disturbances from roughness of the path;

- block cushioning the harvester, taking into account its mass-dimensional parameters; 
- block output of simulation results.

The road surface profile (imitation of the background of stubble of spike crops) is used as an input signal from the methodology for modeling the profile of the road surface and the mass-dimensional parameters of the harvester. The output is:

- vibration velocity, vibration acceleration, movement on the operator's seat;

- frequency at a given section of the harvester movement.

All cushioning masses are reduced to the center of mass of the harvester. The harvester under consideration with a productivity of $1 \mathrm{~kg} / \mathrm{s}$ has a mass distribution scheme along the axes: $80 / 20$ (2035 kg falls on the front axle and $525 \mathrm{~kg}$ on the rear), while the reduced mass of the cushioning part of the combine is $2200 \mathrm{~kg}$.

The results of computer simulation of the cushioning system of the harvester for vibration displacement, vibration velocity and vibration acceleration are presented in Figures 2, 3 and 4.

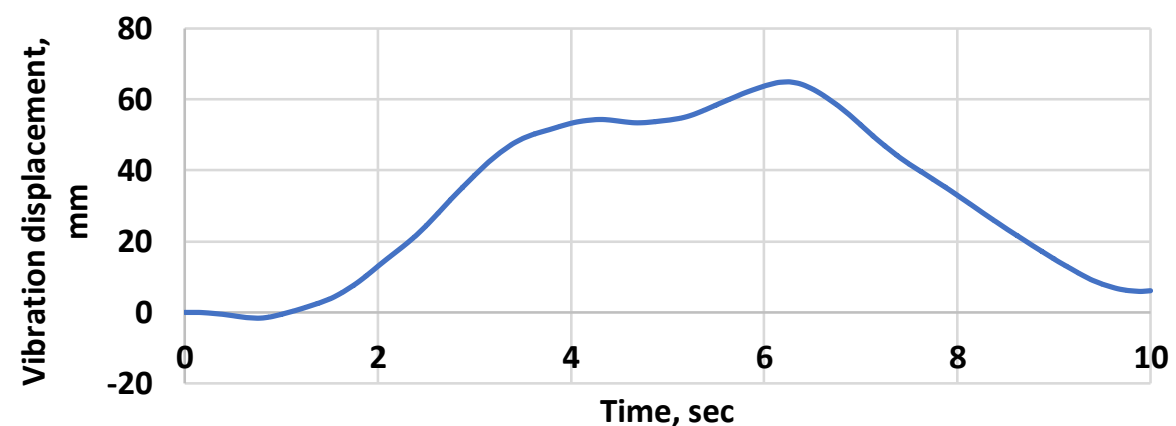

Fig. 2. The results of computer simulation of vibrational displacement of the center of mass of the cushioning part of the harvester.

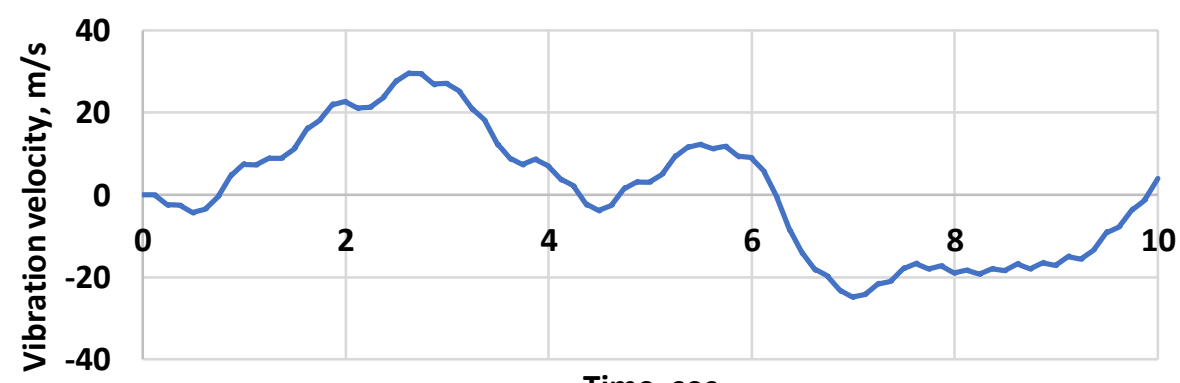

Time, sec

Fig. 3. The results of computer simulation of the vibration velocity of the center of mass of the cushioning part of the harvester. 


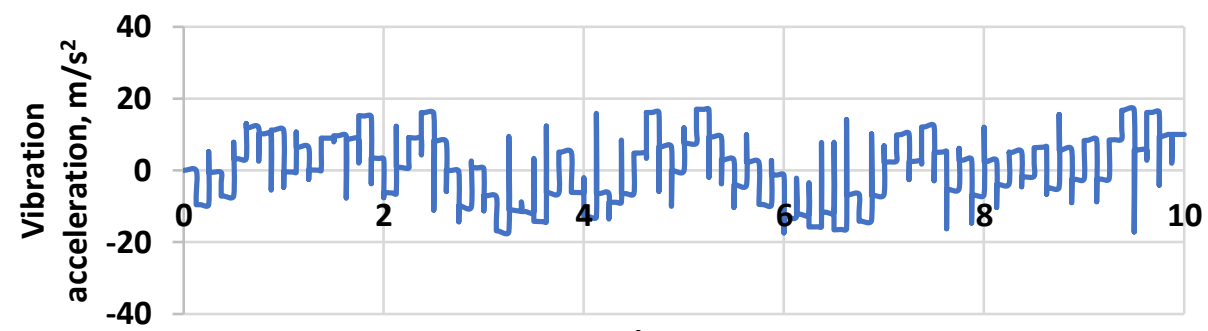

Time, sec

Fig. 4. The results of computer simulation of vibration acceleration of the center of mass of the cushioning part of the harvester.

\subsection{Simulation of directional stability and turning implementation}

Directional stability is one of the key indicators for controlling an unmanned mobile object moving over rough terrain and on loose soil with alternating lateral impact on the steering wheel of the harvester.

Directional stability - the ability of the system to hold a mobile object within a given trajectory for various driving modes (acceleration, braking, driving in a straight section, in turns and when rolling freely).

Table 1. The source data for modeling the processes of directional stability

\begin{tabular}{|c|c|}
\hline Maximum angle of rotation of the harvester, deg & 74 \\
\hline Steering cylinder stroke, mm & 230 \\
\hline Diameter of the piston of the steering cylinder, mm & 40 \\
\hline Volume of the piston of the steering cylinder, 1 & 0.289 \\
\hline Pump productivity, $\mathrm{cm}^{3} / \mathrm{rev}$ & 398 \\
\hline Maximum speed of the hydraulic motor, rpm & 225 \\
\hline $\begin{array}{c}\text { Pump performance at the maximum speed of rotation of the } \\
\text { electric motor, } 1 / \mathrm{s}\end{array}$ & 1.49 \\
\hline $\begin{array}{c}\text { Pump performance at maximum speed of the electric motor with } \\
\text { the declared volume of the piston of the steering wheel, deg/s }\end{array}$ & 383 \\
\hline Velocity of the harvester, $\mathrm{m} / \mathrm{s}$ & 1.79 \\
\hline
\end{tabular}

When driving across the field, the combine deviates from the given trajectory due to external forces acting on it (roughnesses in the road, various tire pressures, and structural problems with the combine).

The task of the directional stability system is to return the combine to the path initially set by the operator by adjusting its movement, this is especially important when making a turn.

The task of adjusting the movement is reduced to assessing the accumulation of deviations from the course and changing the angle of the wheel rotation towards the harvester returning to the original motion path with subsequent stabilization of the result.

The most common reason for the deviation of the harvester from the course is the profile of the road surface and the pressure difference in the wheels of the harvester. The maximum deviation of the harvester from the course should not exceed $10 \mathrm{~cm}$.

Calculation of directional stability is simple according to the following algorithm:

We introduce the resulting motion function $r(t)$, which is determined by the formula:

$$
r(t)=\frac{1}{(f(t)+y(t)+k(t-1)) \times k_{\text {corr }}}
$$

where, $f(t)$ - planned direction of movement; 
$y(t)$ - external disturbance function, which is obtained by generating the random function in the Matlab/Simulink software package (range $\pm 25 \mathrm{~mm}$ );

$k(t)$-correction function;

$k_{\text {corr }}$ - correction coefficient depending on the mass-dimensional parameters of the system and on the performance of the equipment.

To simplify, the mass-dimensional parameters of the system were not taken into account due to the low influence on the value of the correction coefficient.

The correction coefficient is determined by the formula:

$$
\mathrm{k}_{\mathrm{corr}}=\mathrm{A} \times \mathrm{c} \times \mathrm{V} \text {, }
$$

where, $\mathrm{V}$ - volume of the cylinder of the turning system;

A - performance of the hydraulic pump;

$\mathrm{C}$ - participation coefficient of the hydraulic pump in the turning mechanism.

The signal is adjusted in each quantum (step) of the process implementation time. The adjustment step is the integration step in the Matlab/Simulink software package.

The model of the controller for adjusting the movement of the harvester is presented in Figure 5.

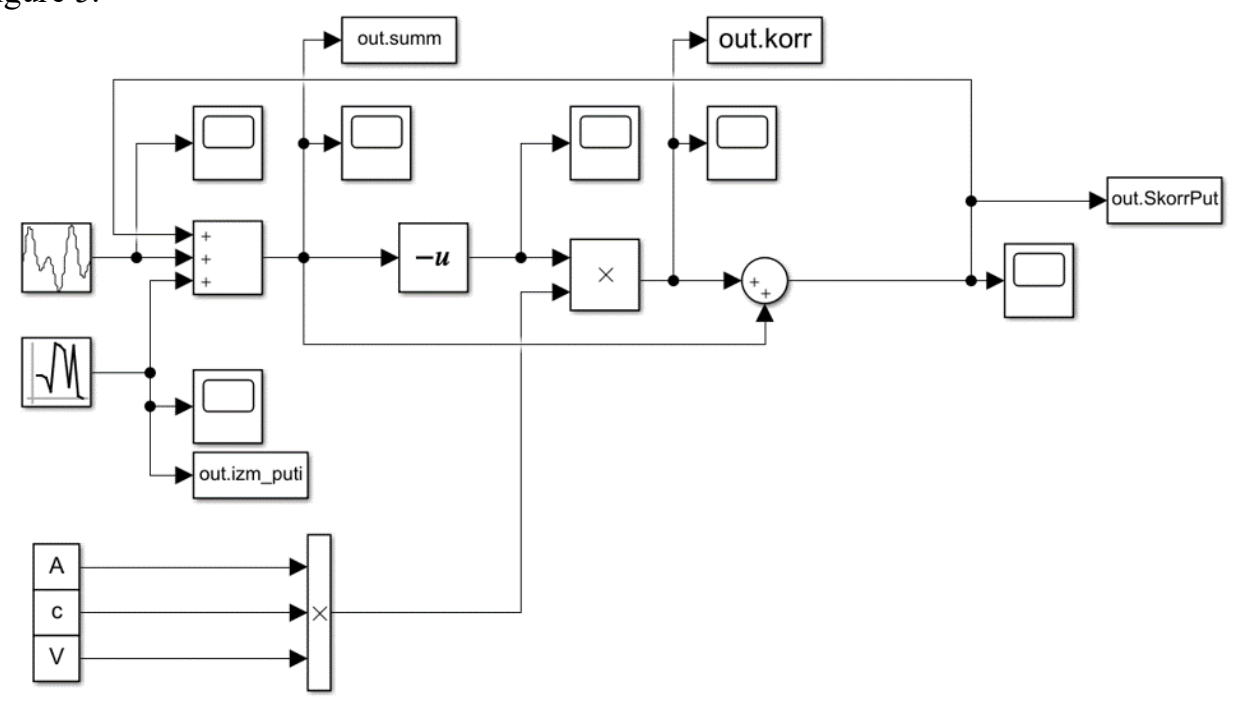

Fig. 5. The model of the controller adjusting the movement of the harvester.

The model consists of 4 blocks:

- block input path of the harvester;

- unit for generation the random deviation from course;

- unit for calculating the correction signal taking into account the design parameters of the harvester;

- result output block.

The result of the generation of an external disturbance is shown in Fig. 6, the correction signal taking into account the hydraulic pump performance is presented in Fig. 7. 


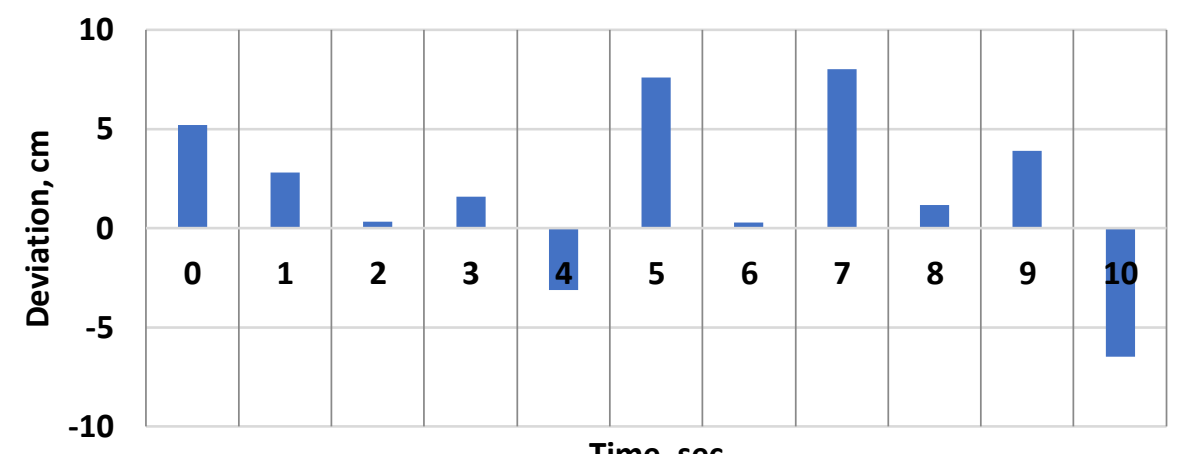

Time, sec

Fig. 6. The external disturbance affecting the deflection of the harvester.

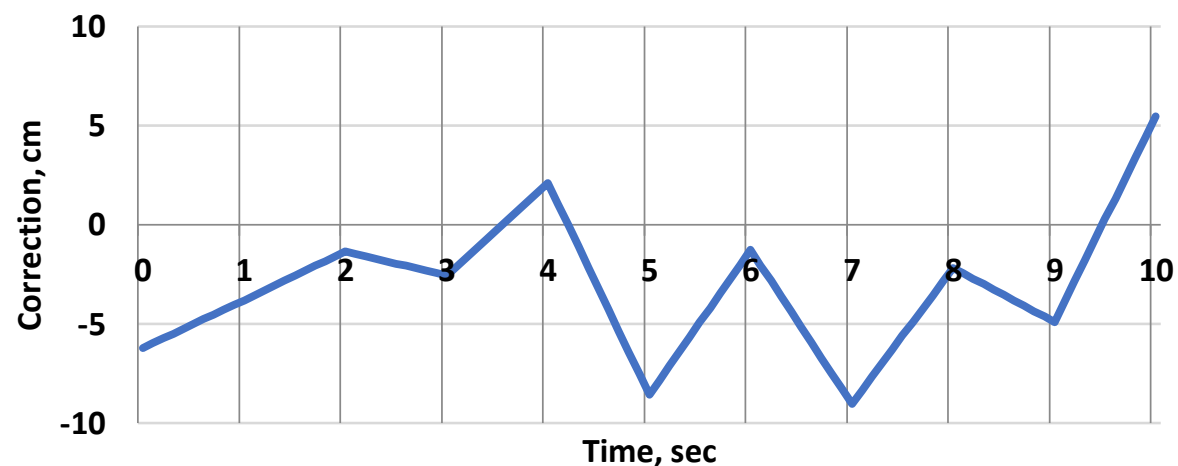

Fig. 7. Tre correction signal of the harvester motion control system.

Summing up the deviation value and the correction signal of the control system, an adjusted course of harvester movement is obtained, taking into account the design features of the combine. The simulation result is presented below in Figure 8.

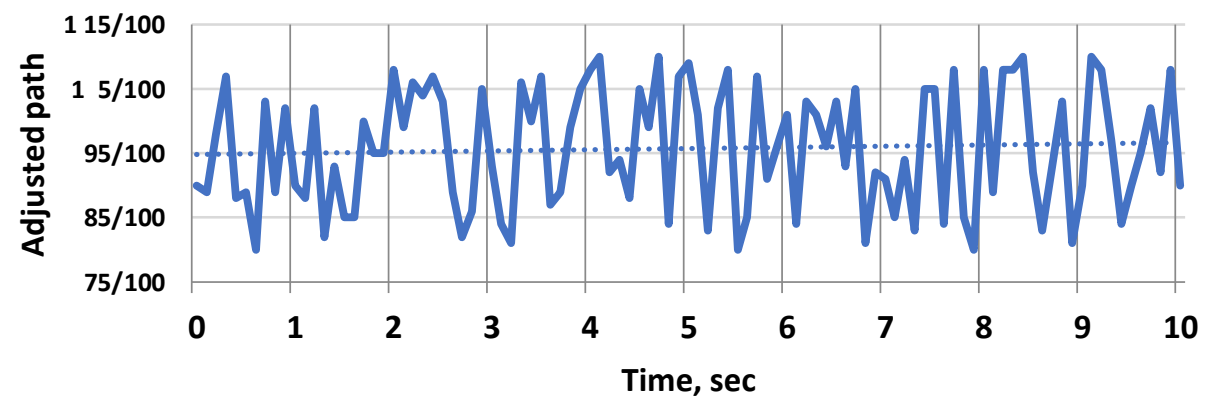

Fig. 8. The adjusted path

\section{Conclusions}

Based on the analysis of the operation of the control system of an unmanned combine 
harvester and the calculation of dynamic movement processes, the maximum values of vibration displacements, vibration velocities, and acceleration were obtained. The vibratory frequency of the harvester was determined when moving along an imitation background the stubble of the spike crops, as well as the values of the maximum deviation of the harvester from a given course of movement.

Based on the results of computer simulation, the parameters of vibration displacement, vibration velocity and acceleration, as well as vibration frequency indicators on the operator's seat are determined. As can be seen from the graphs, the maximum value of vibration frequency is $3.67 \mathrm{~Hz}$, and the difference in vibration acceleration values is 34.15 $\mathrm{m} / \mathrm{s}^{2}$, which corresponds to the safety requirements and technical specifications issued by the customer of the development.

A comparison of the simulation results of the initial and corrected direction of movement of the harvester shows that the maximum deviation from the planned path is a maximum of $10 \%$, which is within acceptable limits. The maximum value of the deviation of the harvester from the course does not exceed $5 \mathrm{~cm}$, which is the permissible accuracy of providing directional stability on the models.

\section{Acknowledgement}

The studies were conducted as part of the Federal Targeted Program for the Grant - Agreement of December 6, 2019 No. 075-15-2019-1692, Ministry of Education and Science of Russia.

\section{References}

1. R. Parray, I. Mani et al. Indian Journal of Agricultural Sciences, 86, 167-172 (2016).

2. M. Levin, A. Degani. Computers and Electronics in Agriculture, 166, 104987 (2019).DOI: 10.1016/j.compag.2019.104987

3. S.N. Subhashree, C. Igathinathane. Biosystems engineering, 187, 185-200 (2019) DOI: 10.1016/j.biosystemseng.2019.08.018

4. D.A. Crolla, A.S.A. El-Razaz. Journal of Terramechanics, 24(3), 199-225 (1987).DOI: 10.1016/0022-4898(87)90041-3

5. I. Melikov, V. Kravchenko, et al. IOP Conference Series: Earth and Environmental Science, 403(1), 012126 (2019) DOI: 10.1088/1755-1315/403/1/012126

6. P. Li, H. Xu. Mathematical Problems in Engineering, 6713231 (2019) DOI: $10.1155 / 2019 / 6713231$

7. F.B.F.D. Lima, M.A.F.D. Silva et al. Engenharia Agrícola, 37(6), 1171-1182 (2017) DOI: 10.1590/1809-4430-eng.agric.v37n6p1171-1182/2017

8. S. Mileiko, T. Bunnam et al. Philosophical Transactions of the Royal Society A: Mathematical, Physical and Engineering Sciences, 378(2164), 20190166 (2019) DOI: 10.1098/rsta.2019.0166

9. O.C. Barawid, A. Mizushima et al. Biosystems Engineering, 96(2), 139-149 (2007) DOI: 10.1016/j.biosystemseng.2006.10.012

10. M.A. Momin, T.E. Grift et al. Precision Agriculture, 20(5), 896-910 (2018) DOI: 10.1007/s11119-018-9621-2

11. A. Tahouni, M. Mirzaei, B. Najjari. Journal of Automobile Engineering, 095440701984885 (2019) DOI: 10.1177/0954407019848858

12. J. Chen, X. Ning et al. Applied Engineering in Agriculture, 33(1), 15-22 (2017) DOI: 10.13031/aea.11299 
13. R.F.G. Baldo, P.S.G. Magalhães. Ciência Rural, 42(2), 298-304 (2012) DOI: 10.1590/s0103-84782012005000001

14. P. He, J. Li. Applied Soft Computing, 77, 387-398 (2019) DOI: 10.1016/j.asoc.2019.01.040

15. J. Mahalakshmi, K. Kuppusamy et al. Emerging Technologies for Agriculture and Environment, 39-52 (2019) DOI: 10.1007/978-981-13-7968-0_4

16. Y. Xie, A. Alleyne. Journal of Dynamic Systems, Measurement, and Control, 136(5), 051006 (2014) DOI: 10.1115/1.4027157

17. Q. Chen, C. Liao, A. Ouyang et al. International Journal of Electric and Hybrid Vehicles, 8(3), 242 (2016) DOI: 10.1504/ijehv.2016.080024

18. A. George, D. Moline, J. Wagner. Renewable Energy, 146, 2659-2667 (2019) DOI: 10.1016/j.renene.2019.08.106

19. S. Senkevich, V. Duryagina et al. Advances in Intelligent Systems and Computing, 1072, 204-213 (2020) DOI: 10.1007/978-3-030-33585-4_20 